\title{
First Implementation of Burrowing Motions in Dual-Reciprocating Drilling Using an Integrated Actuation Mechanism
}

\author{
Craig Pitcher ${ }^{\mathrm{a}, *}$, Yang Gao ${ }^{\mathrm{a}}$ \\ ${ }^{a}$ Surrey Space Centre, University of Surrey, Guildford, GU2 7XH, UK
}

\begin{abstract}
The dual-reciprocating drill (DRD) is a biologically-inspired low-mass alternative to traditional drilling techniques, using backwards-facing teethed halves to grip the surrounding substrate, generating a traction force that reduces the required overhead penetration force. Previous experiments using a proof-of-concept test bench have provided evidence as to the significant role of sideways movements and lateral forces in improving drilling performance. The system is also progressing to a first system prototype concept, in which an actuation mechanism is integrated within the drill heads. To experimentally determine the effect of lateral motions, a new internal actuation mechanism was developed to allow the inclusion of controlled sideways movements, resulting in the creation of the circular and diagonal burrowing motions. This paper presents an investigation into the performance of the reciprocation and burrowing motions by testing them in a planetary regolith simulant. Analysis of force sensor measurements has shown a relationship between the penetration and traction forces and the internal friction of the mechanism and depth achieved. These tests have also experimentally demonstrated the benefit of lateral motions in drilling performance, with both the burrowing mechanisms and drilling tests performed at an angle able to penetrate further than traditional vertical reciprocation, leading to the proposition of new burrowing and diagonal drilling mechanics. From this, a new fully integrated system prototype can be developed which incorporates lateral motions that
\end{abstract}

${ }^{*}$ Corresponding author. Address: Surrey Space Centre, University of Surrey, Guildford, GU2 7XH, UK. Tel: +447816 184142 .

Email address: c.pitcher@surrey.ac.uk (Craig Pitcher) 
can optimise the drilling performance.

Keywords: dual-reciprocating drilling, burrowing, lateral motion, integrated actuation mechanism, diagonal drilling,

\section{Introduction}

Accessing the subsurface of planetary bodies is one of the key aims of extraterrestrial exploration, as it plays a critical role both in the search for life and resources and for furthering our understanding of the formation of bodies in the solar system. The rotary and rotary-percussive terrestrial drilling techniques have been used in past missions such as Apollo as well as for those currently in development, such as ExoMars (Re et al, 2008). However, rotary drills require the use of large masses to provide the overhead forces needed to push the drill into the substrate, while the rotary-percussive drills are complex, heavy systems. As mass is one of the main drivers of a mission, low mass alternatives are being considered. Ultrasonic percussive drills can be used to penetrate hard rocks to shallow depths (Badescu et al, 2007), while self-penetrating percussive moles such as that being used for the InSight mission have been designed to penetrate regolith to depths of at least $3 \mathrm{~m}$ (Grygorczuk et al, 2016).

By taking inspiration from nature, biologically-inspired drilling solutions have been proposed as alternatives to conventional methods (Menon et al, 2006). One such design is the Dual-Reciprocating Drill (DRD) technique, inspired by the ovipositor of the sirex noctilio, or wood wasp. By reciprocating two halves lined with backwards-facing teeth in opposition to one another, the ovipositor is able to burrow into wood in order to lay its eggs. The traction force generated by these teeth, which engage with and grip the surrounding substrate and resist being pulled upwards, is converted to a compressive force in the penetrating half (Vincent and King, 1995). As the forces are generated within the two halves, the drilling motion is achieved with almost no external forces. As such a system would greatly reduce the need for masses required to produce overhead forces, this technique shows great promise in planetary exploration applications.

An initial proof-of-concept design of the $\mathrm{DRD}$, originally envisioned to be used as part of a planetary micro-penetrator (Gao et al, 2007a), demonstrated its potential for drilling in low-strength rocks, with energy requirements comparable to other drilling techniques, and demonstrated a potential 
sample collection method (Gao et al, 2007b). From this, a range of experiments have been performed with the DRD using a custom-built test bench, examining the effects of operational parameters, the regolith preparation methods (Gouache et al, 2011a) and the drill head design (Pitcher and Gao, 2015). These have demonstrated the ability of the DRD to both drill down to depths of over a metre and reduce the overhead force required. However, the technique has exhibited high levels of slippage, caused by the failure of the teeth to properly grip the substrate. Consequently, the presence of lateral movements of the drill head has been seen to have a much more significant role in improving drilling performance than the traction force generated by the teeth.

The design of the DRD is also evolving to convert the test bench actuation mechanism into a compact system prototype. Trade-off studies performed in (Frame, 2012) resulted in the proposition and design of an internal actuation system architecture integrated within the drill heads, though until this point a completed mechanism has yet to be built and tested.

The work presented here aims to further investigate the drilling motion of the DRD by integrating controlled lateral motions into the traditional reciprocating design, allowing an experimental confirmation of the benefits of these sideways movements, and to what extent this has on improving the drilling performance. This will be achieved by evolving the internal actuation system prototype concept to enable both the creation of the original reciprocation and the new combined lateral-vertical motions. This will provide a study of drilling motions never before created, as well as furthering the design evolution of the DRD by demonstrating the first construction and testing of an integrated actuated mechanism.

\section{Importance of Lateral Forces}

Lateral movements of the drill heads were initially observed in the first experimentation of the DRD in regolith, with slight sideways displacements seen during drilling (Gouache et al, 2011a). This was theorised to be caused by the surrounding regolith applying non-vertical forces, due to the conical shape of the drill heads as they are pushed into it, as shown in Figure 1 .

The effects of these sideways movements were discovered in the subsequent mono-block drill head (MDH) experiments. A single solid drill head was reciprocated under controlled operational conditions with a hydraulic ram, with the penetration and traction forces measured using a force sensor 

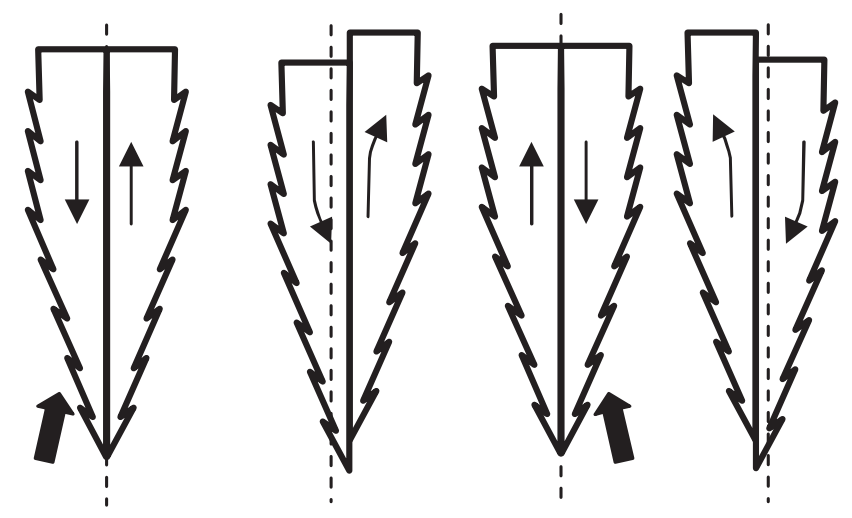

Figure 1: Non-vertical motion (thin arrows) caused by the force applied by the regolith (thick arrows) to the asymmetrical drill head (Gouache et al, 2011a)

test bench (Gouache et al, 2011b). A major conclusion from these experiments was that the traction force generated by the backwards-facing teeth was one to two orders of magnitude lower than the penetration force. As such, the additional penetration force produced by the traction created by the receding drill half is not enough to account for the added drilling depth of DRD compared to static penetration.

The lateral movements observed in the DRD are absent in the MDH, due to the latter's axial symmetry and rigid test-rig. It was proposed that the lateral movements of the DRD assist penetration by minimizing the compression of regolith in front of the drill tip. An analytical estimation calculated that the lateral forces generated by these sideways movements were at least 0.1 times the required penetration force, or more than an order of magnitude higher than the traction force. This confirmed that lateral forces play a much more significant role in the performance of DRD.

\subsection{Drill Stem Bending}

Another series of experiments investigating the effects of drill head design on depth achieved experienced a similar phenomenon. As a result of using a long, flexible drill stem to allow drilling to depths of up to $800 \mathrm{~mm}$, bending of the stem and/or drill heads occurred on numerous occasions. While slight bending generally did not affect results, significant bending as shown in Figure 2, gave very different depth profiles to those produced when the drill stem remained straight (Pitcher and Gao, 2015).

This significant bending would result in an increase in depth reached 

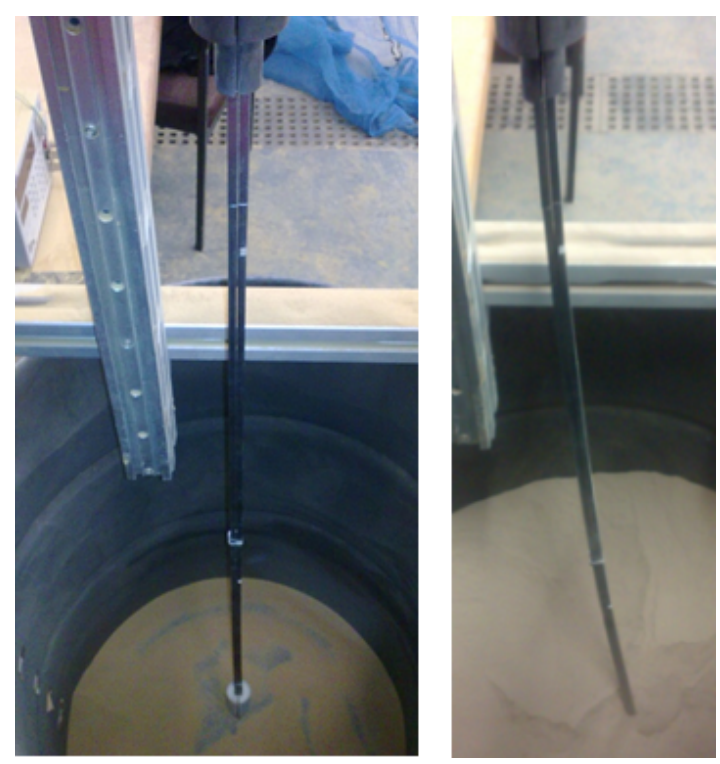

Figure 2: Comparison of the drill stem when resting vertically, and after it has bent during an experiment.

and overall penetration speed. Often the depth profiles of the straight and bent runs would be broadly similar until a certain point, as shown in the example given in Figure 3. Here, both runs reach 500mm at approximately the same time, after which the bent drill continues on to the maximum depth achievable with this set-up, well beyond the final depth reached by the straight run.

From the behaviour exhibited by these experiments, it is proposed that the increase in performance seen with the bent drill stem is due to the direction the regolith is being compacted. The principle of DRD requires the regolith interacting with the teeth to resist the upwards motion of the receding drill half, with resistance provided by the surrounding regolith. When drilling vertically, this is given by the small volume of already sheared regolith directly above the drill head, thus providing only a small vertical resistance. When drilling at an angle, and thus producing lateral movements, the drill is also attempting to move the regolith horizontally into the surrounding regolith, which is able to produce a large horizontal resistance. This results in a larger overall tensile force, reducing slippage and allowing the drill to penetrate further.

From these results it is clear that lateral movements of the drill heads 

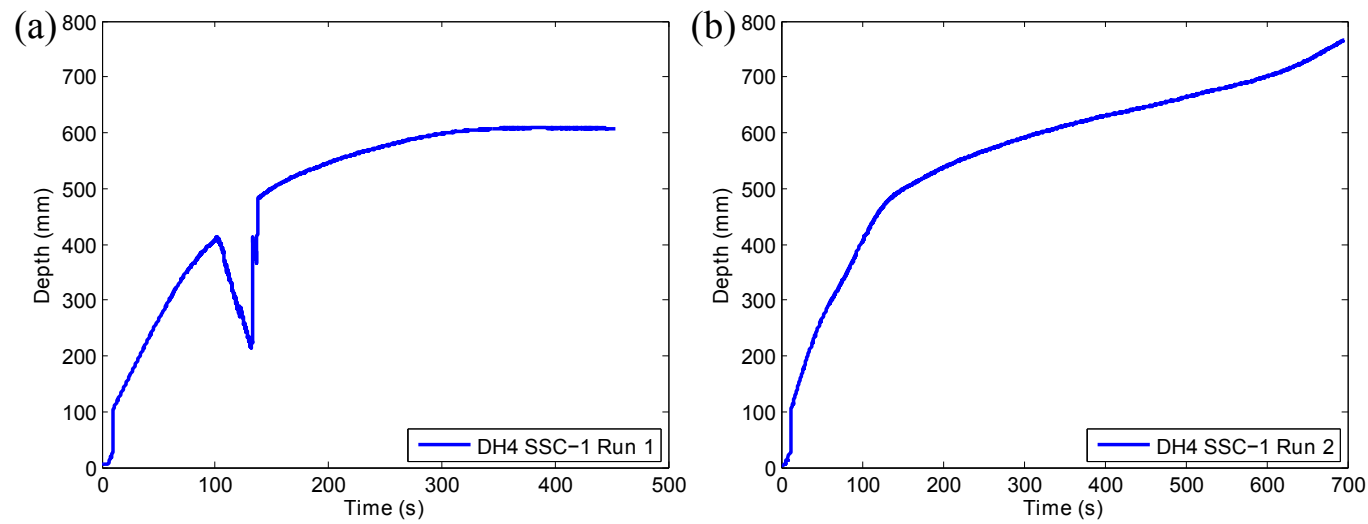

Figure 3: Depth profiles of drill head 4 in regolith with a straight (a) and bent (b) drill stem.

play a major role in increasing the penetration performance of DRD. To confirm these findings, and to determine the extent as to which performance is improved by these movements, active control of the lateral motion is required.

\section{Dual Complex Motion Mechanism Design}

The trade-off studies performed to create the first design of a fully integrated DRD actuation system resulted in the implementation of a simple quad cam drive system. This links the rotary motion of a conventional motor drive via a shaft and bevel transfer box to two pairs of rotary cams. The cams are connected to a drive rail coupling, converting the rotary motion to linear reciprocation. A fully integrated system was designed, shown in Figure 4 (a), incorporating the mechanism, payload bays and motor within a single module, and a partial model showing the cam-drive mechanism was built, seen in Figure 4 (b). The drill would then be deployed using a bistable composite mechanism, which unrolls as the drill descends and can produce a stable overhead force of up to 500N (Gao et al, 2015).

The original integrated design prototype will be used as inspiration for a new mechanism capable of producing vertical-only reciprocation and combined lateral-vertical motions. These are referred to henceforth as simple and complex motions respectively. This new actuation system will be referred to as the Dual Combined Motion Mechanism (DCMM). 

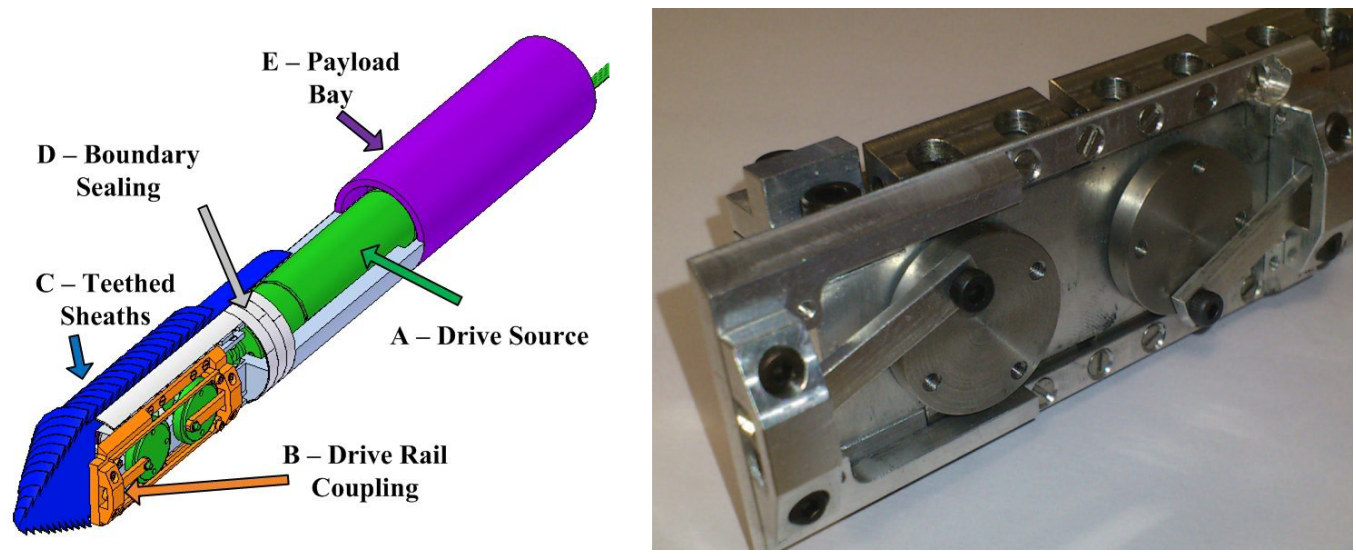

Figure 4: (a) A CAD model an the envisioned integrated DRD system prototype (Gao et al 2015) and (b) the constructed partial model of the quad cam drive system (Frame, 2012 ).

\subsection{Concept Design Trade-off and Selection}

Before the construction of the DCMM could begin, a number of concepts were created. A critical requirement to be met by these concepts regards the complex motion produced. The drill halves must undergo a cycle in which they are pushed horizontally outwards before or during the upwards motion, and are pulled inwards before or during penetration. This will maximise the horizontal force, and by extension the overall tension force, by digging into and increasing the interaction with the regolith during retraction. It will also minimize the penetration force required by reducing the total volume of regolith being penetrated by the drill heads, as shown in Figure 5. The five concept designs that met this requirement were subjected to a trade-off study to determine their overall suitability, detailed in (Pitcher et al, 2015).

\subsection{Quadruple Cam Design}

The trade-off study resulted in the selection of the Quadruple Cam design. This uses the original cam-drive system as shown in Figure 4 for the simple motion, though with only one cam per vertically-reciprocating drive rail. The complex motion is achieved through the use of two additional cams positioned $90^{\circ}$ around the motor shaft, with the corresponding drive rails converting the rotary motion to horizontal reciprocation, as seen in Figure 6.

A major consideration which drove the design of the Quadruple Cam design was the overall size. While length was not strictly constrained, the 


\section{Simple motion Complex motion}
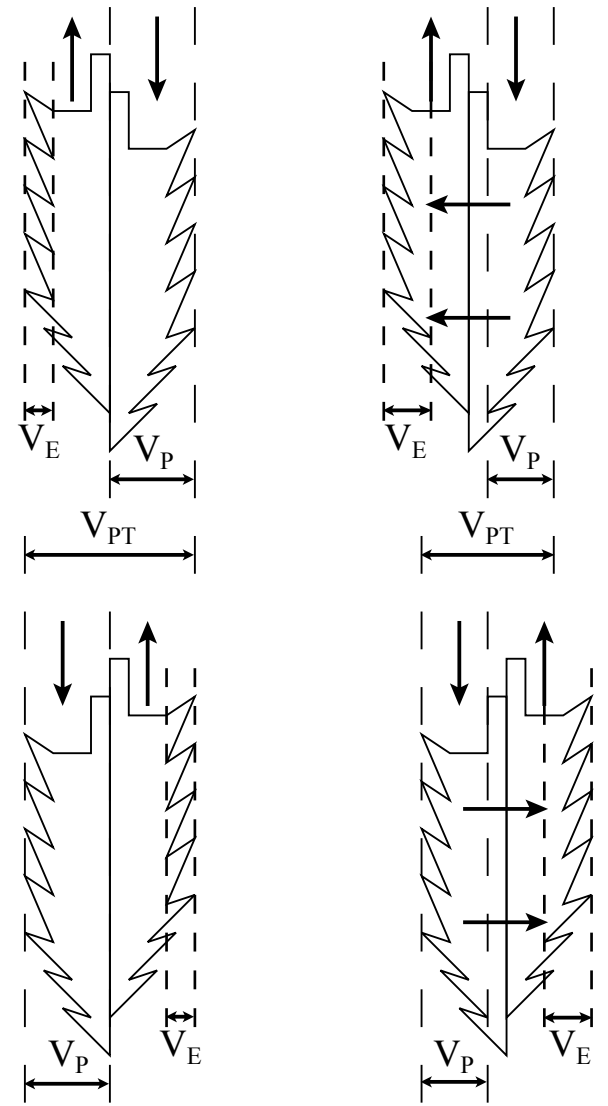

Figure 5: Diagram of the simple and complex motion cycles, demonstrating the volumes of regolith penetrated by each penetrating half $\left(V_{P}\right)$ and in total $\left(V_{P T}\right)$, and the volume of regolith engaged by the teeth for each receding half $\left(V_{E}\right)$.

drill diameter had to be kept as small as possible. Given the complicated design and number of parts involved, the desired diameter of the completed drill was $46 \mathrm{~mm}$ (the same as the largest drill head design in (Pitcher and Gao, 2015), with a limit of $56 \mathrm{~mm}$. As with the original design, the DCMM is actuated by a commercially available off-the-shelf DC motor, with the shaft mechanically linked to two transfer boxes. These consist of three bevel gears; one attached to the motor shaft, and the other two positioned at $90^{\circ}$ and each attached to a cam. The top transfer box drives the vertical rails, and the bottom drives the horizontal rails. Ideally, a single transfer box would have driven all four cams, but spacing issues made this impossible. Additionally, 

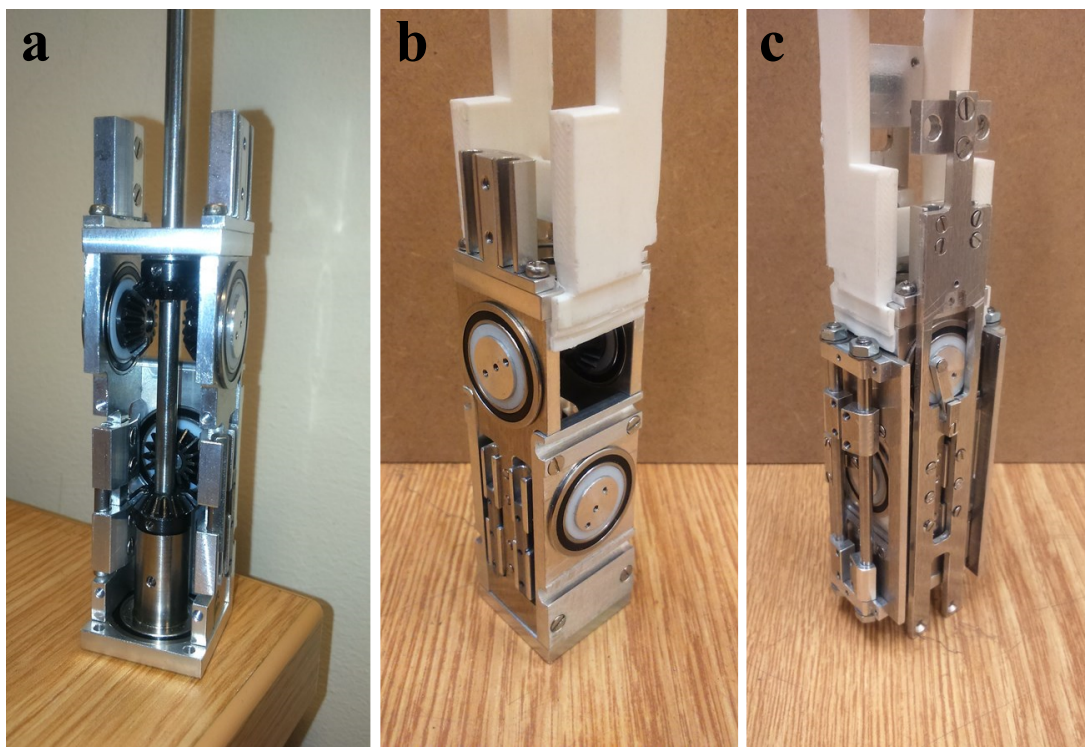

Figure 6: Pictures of (a) the bevel gear transfer boxes, (b) the central holding structure of the DCMM and (c) the vertical and horizontal drive rails.

space and sizing constraints prevented the use of two cams per drill head. The cams and motor shaft are slotted into a central holding structure using ball bearings. The vertical and horizontal drive rails use a series of precision bars that are allowed to slide linearly through guide slots cut into the central hold. Each cam has three holes, allowing reciprocation amplitudes of 1, 2.75 and $4.5 \mathrm{~mm}$.

The drive rails are fixed to the drill heads by two hollow outer shell structures, as shown in Figure 7 (a). This is achieved with a series of connection points consisting of a combination of precision slide bars and linear guide supports. These supports allow the horizontal and vertical linear motions of the drive rails to be converted to complex motion in the outer shells. In the vertical drive rails, the supports are pushed vertically by the slide bars, whilst simultaneously allowing them to freely move horizontally. Multiple connection points per drive rail are used to reduce the friction of this motion and subsequent risk of jamming in each slide bar.

The drill heads were made of three ABS plastic parts fixed together, and attached to connection points in the outer shells. The teeth were given a width of $4 \mathrm{~mm}$ and height of $7 \mathrm{~mm}$, giving the drill heads a total diameter of $54 \mathrm{~mm}$ and a length of $230 \mathrm{~mm}$. For these experiments, only the actuation 
mechanism is required to be held within the drill heads. As a result, the motor is held separately above the drill and attached to a wooden plate. To stop the mechanism rotating around the motor shaft, an additional plastic/wooden holding part is wrapped around the central structure and attached to the plate, shown in Figure 7 (b).
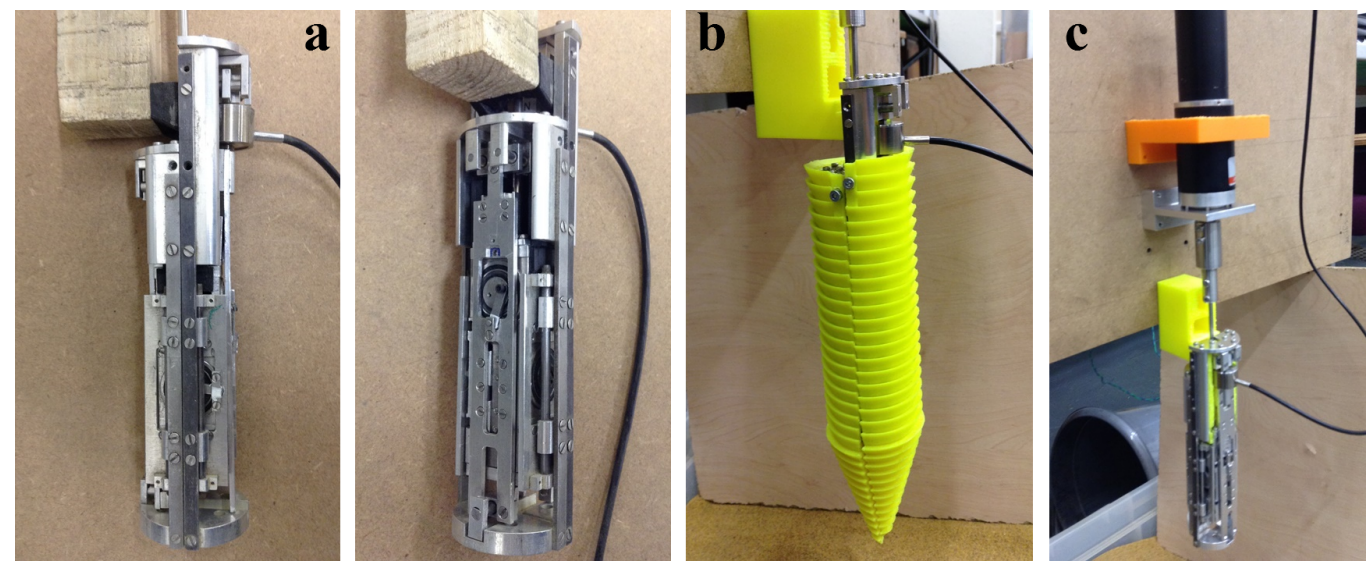

Figure 7: Pictures of (a) the outer shell, (b) the holding attachment and (c) the fully assembled mechanism

\section{Motions Available}

The major advantage of the Quadruple Cam design is that the vertical and horizontal motions are independent from each other; in other words, the amplitude of the vertical reciprocation, $a_{v}$ can be changed without affecting the horizontal reciprocation amplitude, $a_{h}$, and vice versa. An additional benefit of this is that, depending on the set-up of the cam wheels and connecting rods, the mechanism can produce either a circular or diagonal motion. This results in three distinct series of motions that can be examined: simple motion, circular burrowing and diagonal burrowing.

Figure 8 (a) shows the circular motion created when both $a_{h}$ and $a_{v}$ are set at $4.5 \mathrm{~mm}$. Here, the lines trace the paths travelled by the drill heads when viewed from the angle in Figure 7 (a), with their respective central points both positioned at the origin. Due to the piston-like motion of the cam-drive system being non-sinusoidal, the resultant motion is actually a slightly squashed circle. Decreasing both amplitudes equally results in a more regular shape, while reducing only one amplitude, seen in Figure 8 (b), 
results in an elliptical path. As each cam wheel has three amplitudes, this gives a total of nine circular burrowing combinations.

As with the circular burrowing, there are nine amplitude combinations that can be produced for diagonal burrowing by altering the amplitude combinations. A slight difference can be seen with regards to the paths' regularity. Whereas the shape is a straight diagonal line when the amplitudes are equal, a slight curve can be noticed as the amplitudes begin to differ, seen in Figure 8 (d). As these irregularities are slight, any effect on drilling performance will likely be negligible (Pitcher et al, 2015). Along with the three amplitudes available for the simple motion, there are a total of 21 motions that can be produced.

\section{Experimental Plan}

To evaluate the benefits of the burrowing motions compared to simple reciprocation, a test rig was designed to allow the recording of forces experience within the drill head and depths achieved. The wooden plate is attached via dry bearings to guide rails, allowing it to slide vertically with minimal friction. To measure depth, a membrane potentiometer is stuck to one of the rails, with a roller attachment added to the plate that presses into it, similar to the experiments performed in Pitcher and Gao (2015).

The forces experienced within one drill head will also be measured. To do this, a DCM tension-compression load cell, able to measure forces up to $2.5 \mathrm{kN}$, is placed between one of the vertical drive rails and its slide rail connections, as can be seen in Figure 7. In this configuration, all forces experienced between the drive rail and outer shell are transmitted through the sensor. During retraction of the drill head, the resistance of the regolith against the upwards movement of the drive rail is registered as a tension force, while the resistance of the regolith to the downwards penetration of the drive rail results in a compression force. A custom-built amplifier was used to provide a readable range of $848 \mathrm{~N}$ with a resolution of $1.6 \mathrm{~N}$. Both the force and distance readings were recorded using an Arduino Uno.

The experimental approach detailed here is chosen in favour of numerical modelling methods, as previous million-plus particle DEM simulations have had limited success in replicating the forces experienced by the drill heads (Gouache et al, 2011b). However, the data obtained here can potentially be used for the creation of future models able to more accurately simulate the DRD's performance in regolith. 


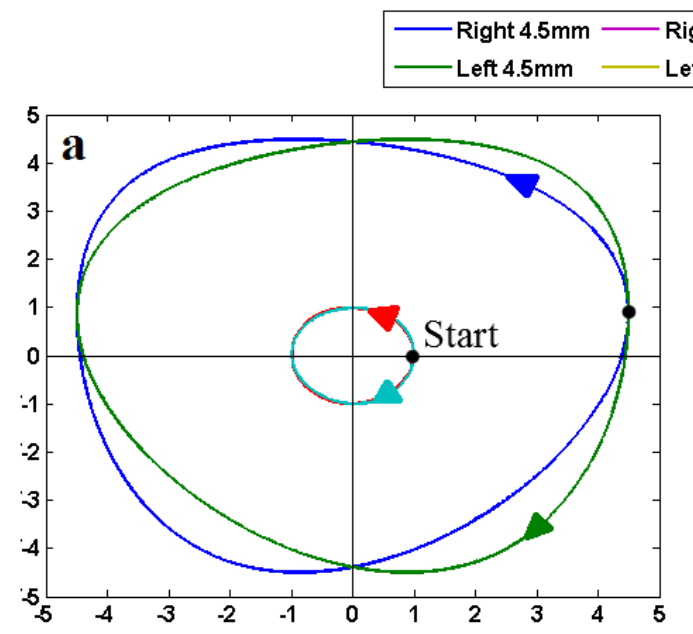

Right $2.75 \mathrm{~mm}-$ Right $1 \mathrm{~mm}$
Left $2.75 \mathrm{~mm}=$ Left $1 \mathrm{~mm}$
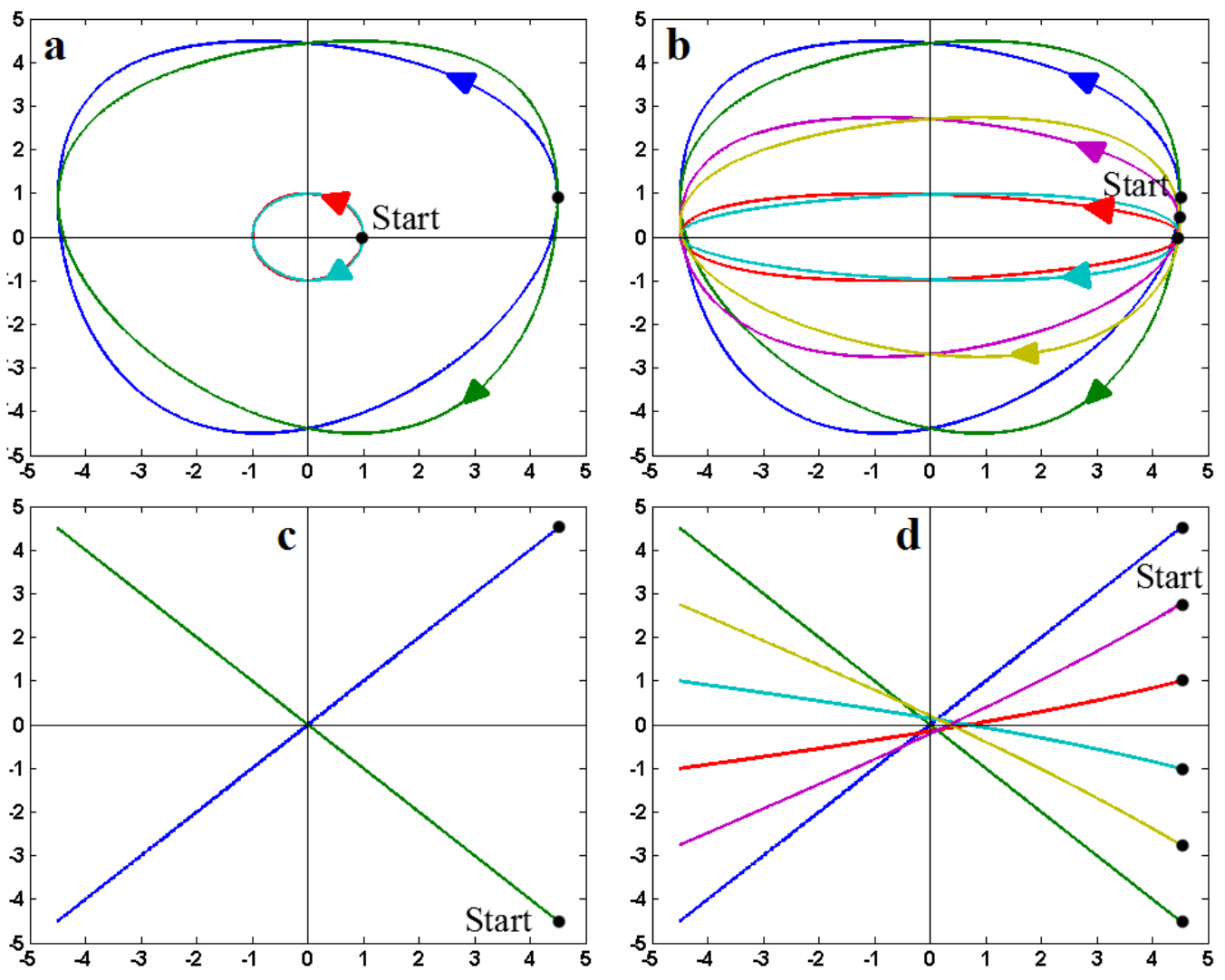

Figure 8: Graphs of the circular (top) and diagonal (bottom) paths travelled by the drill heads with equal $a_{h}$ and $a_{h}$ (left) and with different $a_{v}$ values and a constant $a_{h}$ of $4.5 \mathrm{~mm}$ (right). 
Each drilling motion will be tested twice in the Surrey Space Centre Mars regolith simulant 1 (SSC-1) (Scott and Saaj, 2009), poured in a box $28 \mathrm{~cm}$ deep. SSC-1 is a coarse quartz sand, with particle sizes of $100-1000 \mu \mathrm{m}$, with a density of $1413 \mathrm{kgm}^{-3}$ and a relative density of $7.4 \%$ when poured (Gouache et al, 2011a).

Drilling in regolith presents jamming risks caused by the material's abrasive nature. Regolith particles and dust that are able to enter the drill heads pose a jamming risk to the internal mechanism, given its intricate nature, which worsens significantly for fine-grained regoliths. Effective sealing of the drill heads to prevent this, as would be used in a true system prototype, could not be utilised for this particular mechanism. To enable smooth operation of the mechanism for as long as possible, testing was limited to the coarse SSC-1.

To further extend the longevity of the mechanism as far as possible into the experiments, the simple motion amplitudes were tested first. After this, the circular and diagonal motions were tested together, using all $a_{v}$ values with the $a_{h}$ fixed at $1 \mathrm{~mm}$. This was then repeated with the $a_{h}$ set to $2.75 \mathrm{~mm}$, and once more with the $a_{h}$ at $4.5 \mathrm{~mm}$. Due to factors discussed in Section 8 resulting in unrepresentative results, the simple motion and circular burrowing tests performed with the $a_{h}$ set at $1 \mathrm{~mm}$ were redone.

The reciprocation frequency of the drill is kept constant by using a continuous voltage power supply, with $5 \mathrm{~V}$ equating to a frequency of approximately $1 \mathrm{~Hz}$. The overhead force provided by the test rig was set at $48 \mathrm{~N}$. For each experiment, the sensors begin recording and the rig is held with the drill tip just above the regolith surface. The rig is then released, after which the drilling is stopped after 100s. For each experiment, the drill had reached its final depth within this time. In the analysis of the results from this point, the amplitude combinations for each motion are presented in the shortened form of $x \mathrm{~V} y \mathrm{H}$; for example, a motion labelled as $4.5 \mathrm{~V} 1 \mathrm{H}$ uses a vertical amplitude of $4.5 \mathrm{~mm}$ and a horizontal amplitude of $1 \mathrm{~mm}$.

\section{Force Sensor Measurements}

Measurements with the force sensor were made during free-running, i.e. when the drill is freely in motion whilst held above the regolith, and during drilling, with the compression and tension recorded as negative and positive values respectively. The free-run profile of the $4.5 \mathrm{V0H}$ simple motion, given 
in Figure 9 (a), allows a close-up look at the force values. A very consistent profile can be seen, with the spikes in compression and tension reaching similar maximum values. Figure 9 (b) shows the drilling force-depth profile, and shows that both forces increase with depth, though the increase in compression is noticeably smaller.
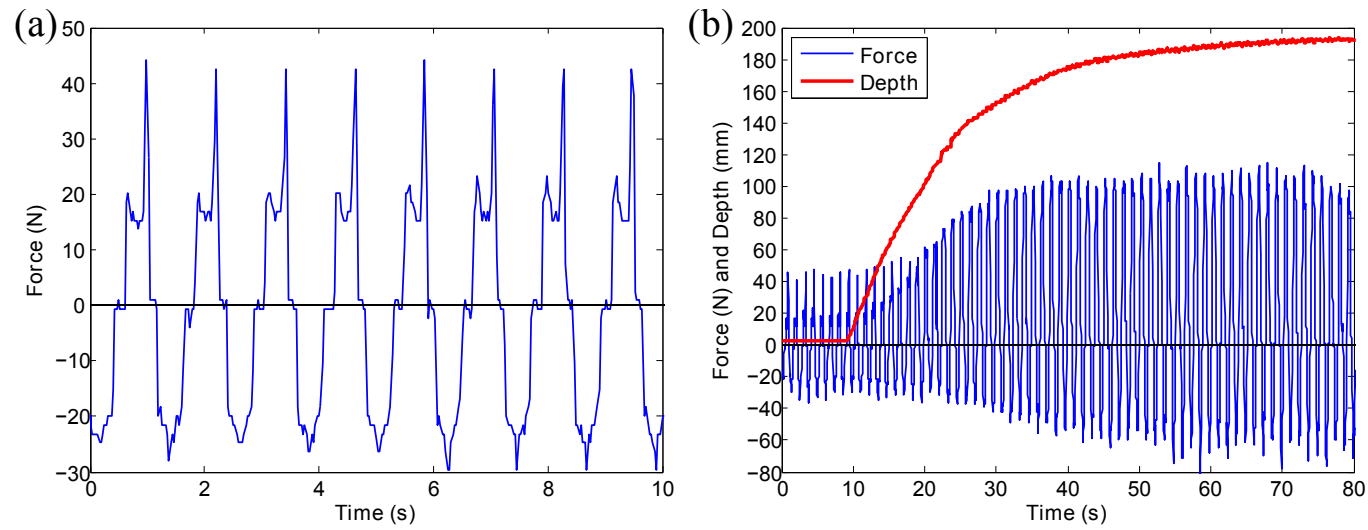

Figure 9: Graphs of the force measurements for the free-run (a) and the combined forcedepth profile for the drilling of the $4.5 \mathrm{~V} 0 \mathrm{H}$ set-up.

The increases in tension and compression forces were compared to the final depths reached for experiment, given in Figure 10. Here, the tension and compression data are separately grouped into their respective $a_{h}$ values. In all cases, it can be seen that both forces increase with depth reached, with the tension, or penetration, forces being larger than the retraction forces. The clearest correlation is seen for the simple motion, when $a_{h}=0$, with the complex motion results all tending to be slightly more scattered. This scattering, and the negative values of some compression measurements, suggests that the measurements of the force sensor may not be especially accurate. Given the combination of the lateral movements and complexity of the mechanism, discrepancies such as this are to be expected. As a result, any further accurate analysis of the forces experienced in the complex motions beyond the generalisations made here is not possible. However, analysis of the forces during simple motion can still be made.

\subsection{Determination of Forces During Reciprocation}

To understand the forces being experienced during a single reciprocation cycle, the starting positions of the drill heads were taken into account. Figure 11 shows the force profiles of two slow cycles given when the drill head 


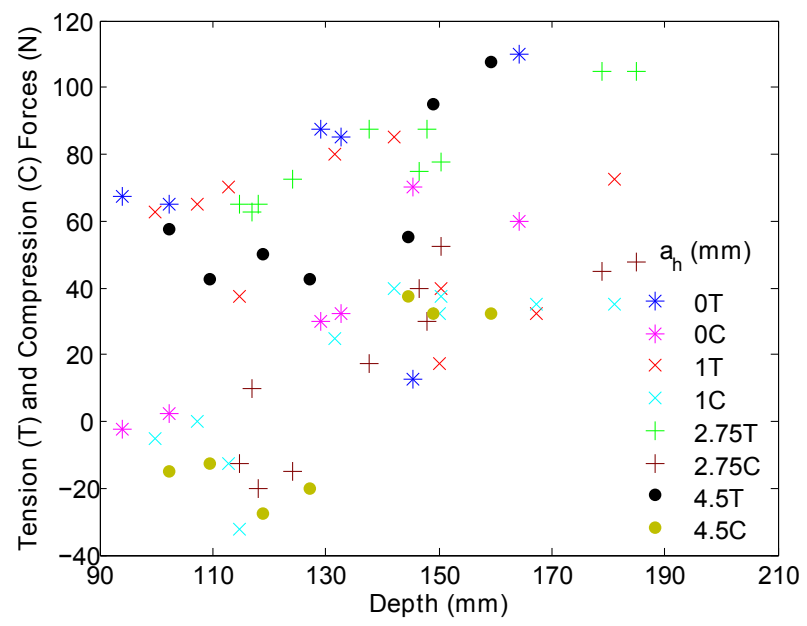

Figure 10: Graph showing the relationship between the final tension and compression values and depths reached for each experiment. The data is grouped into the type of force and their respective $a_{h}$ values.

containing the force sensor is started from the peak retraction and penetration positions. From this, it can be seen that the forces experienced during a reciprocation cycle are independent from the starting position. It also shows that a force is already acting on the sensor before any motion has started, whether it be the compression at the retraction point or tension during penetration. This suggests that much of the force exerted on the sensor is created by resistance from the internal friction of the mechanism, which increases as the drill head moves away from the mid-point.

During free-running, the forces acting on the sensor are created by the motor, $F_{m o t o r}^{\prime}$, and resistance created by internal friction, $F_{\text {int }}$. During drilling, increased current from the motor, as discussed in (Pitcher and Gao, 2015), results in a change in motor force, $F_{\text {motor }}$, while resistance created by the regolith, $F_{\text {res }}$ is also added. The total forces are given in Equation (1).

$$
\begin{gathered}
\text { Free-run: }( \pm) F_{\text {comp } / \text { tens }}=F_{\text {motor }}^{\prime}+F_{\text {int }} \\
\text { Drilling: }( \pm) F_{\text {comp } / \text { tens }}=F_{\text {motor }}+F_{\text {int }}+F_{\text {res }}
\end{gathered}
$$

The contribution of each force to the sensor readings during free-running can be analysed by modelling the reciprocating system as a slider-crank mechanism, as shown in Figure 12. The net work performed on a vertical piston 


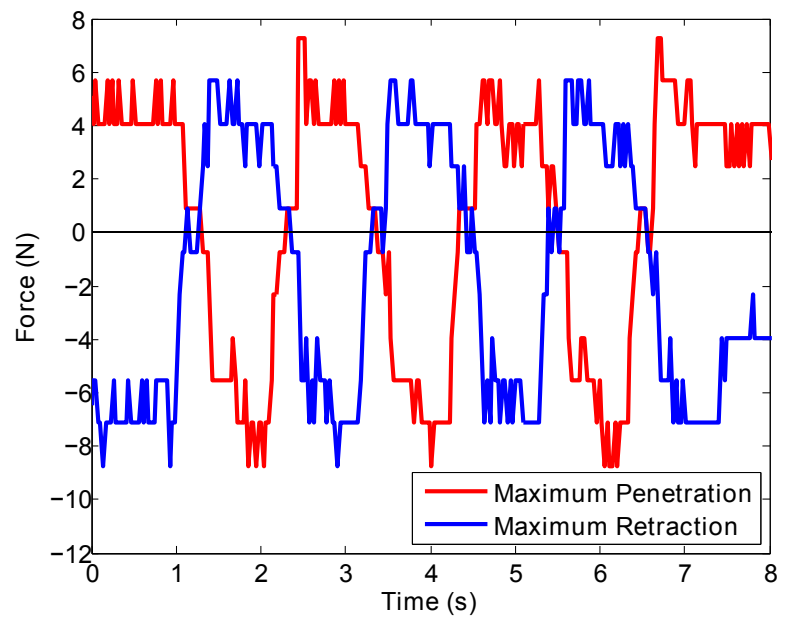

Figure 11: Graph of the force profiles at different starting positions.

engine (Bansal and Brar, 2015) can be rearranged to determine the net force required to operate the crankshaft, given in Equation (2).

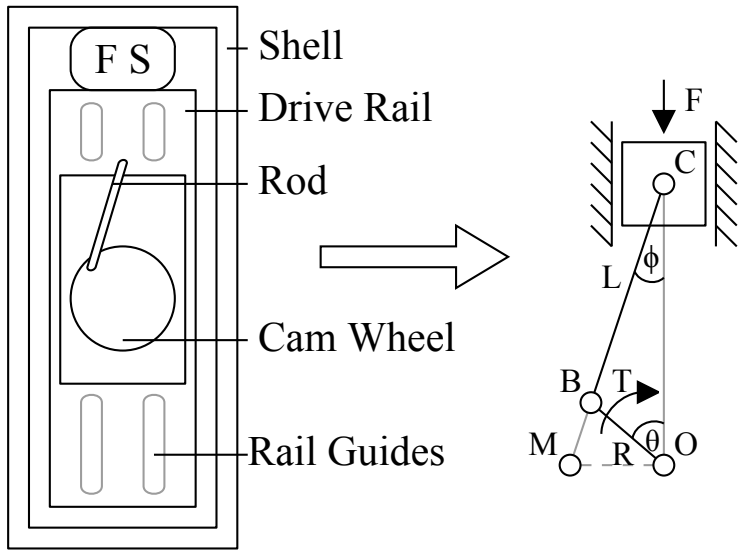

Figure 12: Diagram of the simplified reciprocating drive rail converted to a slider-crank mechanism.

$$
F_{\text {net }}=F_{\text {int }} \pm F_{w}-F_{i}
$$

The weight of the mechanism, $F_{w}$, contributes to the overall effort placed on the crankshaft during the upstroke, and reduces it during the downstroke, hence the positive-negative change. Inertial force, $F_{i}$, given by D'Alembert's 
principle as the body's resistance any change to its velocity, can be analytically calculated. In a frictionless system, these values give the total motor force needed to rotate the crankshaft. Multiplying this by distance $O M$ gives the calculated motor torque, $T_{c}$, required to overcome these forces. The forces measured by the sensor, which include friction, are also converted to torques, $T_{m}$, and the torque cycles with and without friction during one reciprocation cycle are compared in Figure 13 .

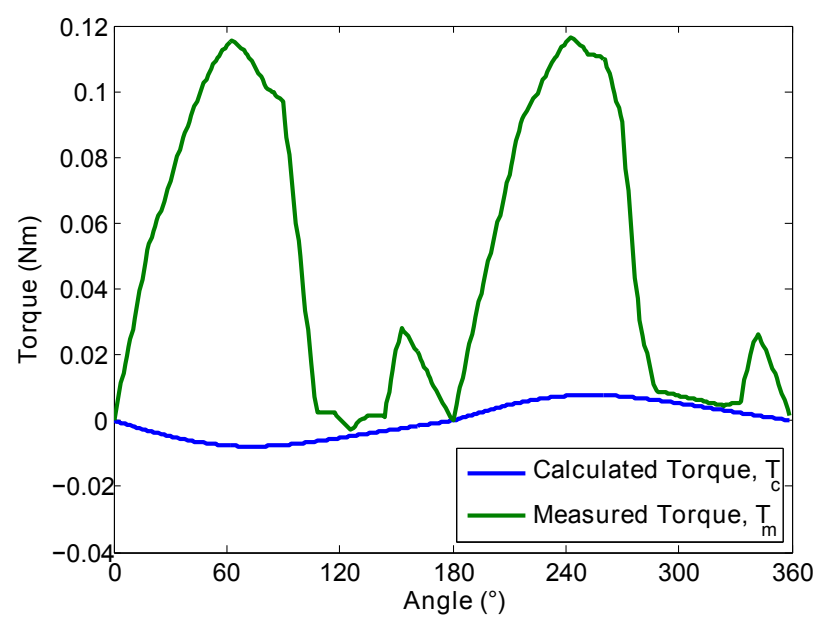

Figure 13: Graph of the calculated and measured torques for the system without and with friction respectively.

From this it can clearly be seen that the measured torque is much larger than the calculated, and as such it can be concluded that the internal friction is significantly the most important force within the mechanism. By assuming that the internal friction values in a reciprocation cycle remain constant for free-running and during drilling, and given the very small motor force values calculated, the increase in forces measured during drilling can be attributed solely to the regolith interaction.

\subsection{Current Analysis}

Further examination of the internal friction can be achieved by determining the current profiles for the DCMM at different stages of construction. Figure 14 shows the averaged motor current measured for the mechanism at increasing stages of completion, from driving only the transfer box and cam wheels to the fully completed system. As parts begin getting added, a clear cyclical pattern of the current, and by extension the friction, emerges, 
which is consistent with the measurements made in Figure 11. It can also be seen that the addition of the drill heads accounts for by far the greatest increase in current. This pattern remains the same throughout the experiments, however the values, in particular those of the full assembly, can show significant increases depending on the set-up of the system, including tightness of the screws, chosen amplitudes, cleanliness of the guide rails, etc. As such, periodic maintenance of the DCMM was critical to avoid jamming and breakages. This is very much a limitation of the mechanism's construction which, as a research tool, has not been subjected to the stringent tolerances and conditions that would be expected from a true system design prototype.

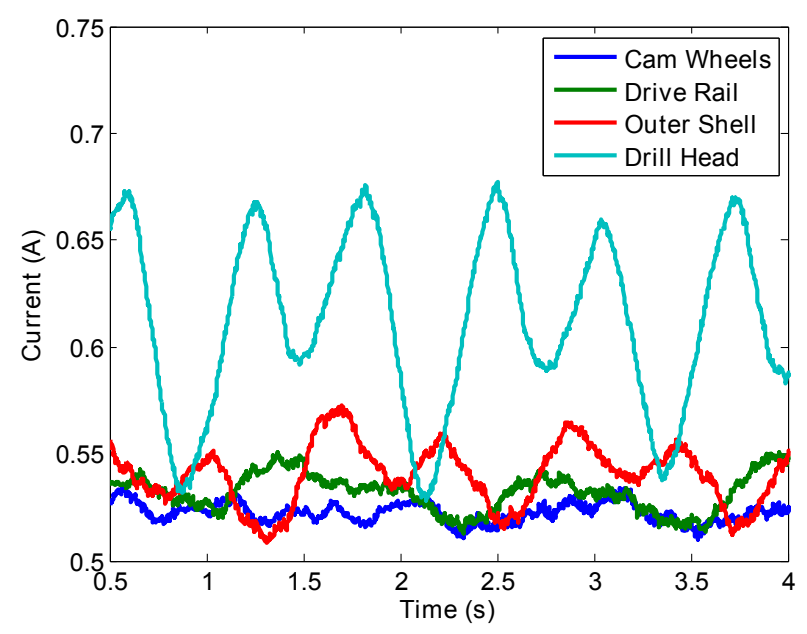

Figure 14: The current readings measured with the internal mechanism in increasing stages of completion.

\section{Depth Analysis}

This section examines the depths achieved by most of the available drilling motions. Severe difficulties in maintaining the drill to run smoothly with the $a_{h}$ set at $4.5 \mathrm{~mm}$, and the strain put on the mechanism when the $a_{v}$ is $4.5 \mathrm{~mm}$, created a significant risk of serious failure, and as such the $4.5 \mathrm{~V} 4.5 \mathrm{H}$ motions were not tested. However clear conclusions can still be drawn despite this omission.

\subsection{Final and Drilling Depths}

The simplest results that can be measured are the final depths, which are given as the total depth achieved after the experimental time of 100 seconds. 
Alternatively, the actual drilling depths can be obtained by excluding the initial penetration caused by the release of the test rig. The initial penetration depth was estimated by dropping the stationary drill into the regolith several times. The depths measured varied from $84.5-98.5 \mathrm{~mm}$, giving a mean of $88.9 \mathrm{~mm}$ and a standard deviation of $6.514 \mathrm{~mm}$. While the initial penetration cannot be accurately measured for each individual experiment, the small standard deviation suggests that the variation of these depths should not have any significant effect on the final and actual depths obtained.

Figure 15 (a) presents the final depths of all experiments, separating both the amplitudes and the circular and diagonal burrowing (CB and DB) motions, with the results obtained from the simple motions also separated. The individual results are given as the scatter points, while the averages of the two results for each motion are represented by the bars. The actual drilling depths can be calculated by substracting $\sim 90 \mathrm{~mm}$ from each value. The most important conclusion that can be drawn from this graph is that the simple motions have the lowest final depths for all $a_{v}$ values, with the exception of the $4.5 \mathrm{~V} 1 \mathrm{H} \mathrm{CB}$. Additionally, there is a general trend of increasing depth achieved with increasing $a_{h}$. The major exception to this is the $1 \mathrm{~V} 4.5 \mathrm{H} \mathrm{DB}$, which is significantly smaller, however given the trends demonstrated by the other motions, this can be considered an anomaly. By disregarding this, another trend that can be seen is that the DB motions for each $a_{h}$ tend to reach slightly greater depths than their equivalent $\mathrm{CB}$ motions.

Figure 15 (b) gives the actual depth gains each complex motion achieves from their respective simple motion for each $a_{v}$ setting. Here it can be seen that the overall gains fall within the same range, with no obvious trend between each $a_{v}$ group. A number of the $a_{h}$ depths also are broadly similar to each other for each $a_{v}$ value. This suggests that the depth gains from simple motion created by the lateral movements increase as the $a_{h}$ gets larger, and remain fairly constant for each $a_{v}$.

The significance of these trends can be examined by looking at the percentage increase in actual drilling depth each complex motion produces from the simple motion, given in Figure 16. Given that the drilling depth of the simple motion when $a_{v}=1 \mathrm{~mm}$ was only $\sim 10 \mathrm{~mm}$, the depth gains presented in Figure 15 (b) result in a huge percentage increase, ranging from approximately $50-270 \%$. The percentages for the motions with the $a_{v}$ set at 2.75 and $4.5 \mathrm{~mm}$ are much smaller, with a maximum gain of roughly $50 \%$. Given that the depth gains remain fairly constant with increasing $a_{v}$, this decrease is expected. As such, this shows that the benefit of lateral movements is 

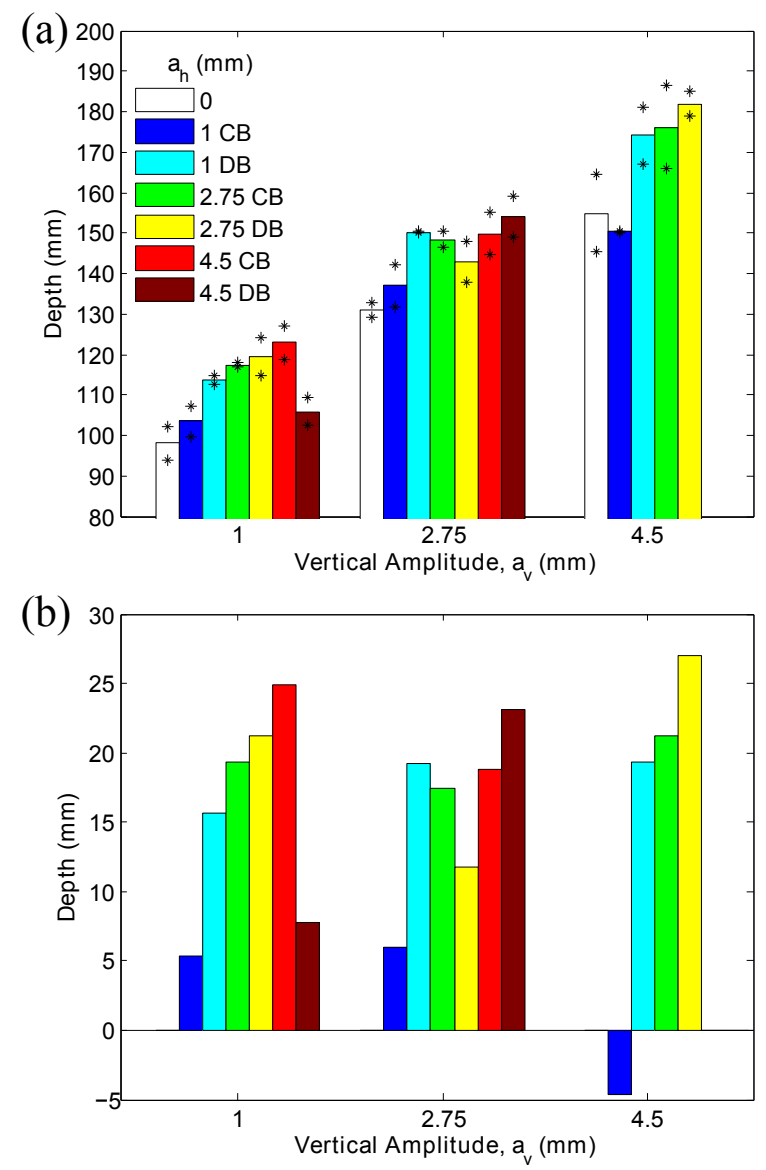

Figure 15: Bar graphs showing (a) the average and individual final depths reached by each motion and (b) the depth gain of the CB and DB from the simple motions. The legend in (a) can be used for both graphs.

most noticeable for motions with lower vertical amplitudes.

\subsection{Examination of Burrowing Mechanics}

The results given here can be compared to the drilling mechanics discussed in Section 3.1. The addition of sideways movements can be seen to improve the drilling performance, and this can be attributed to both the improved gripping and resistance of the regolith and reduced penetration requirement.

However, an observation made during the runs with the larger $a_{h}$ values was the significant reduction of the drill heads' lateral movements once 


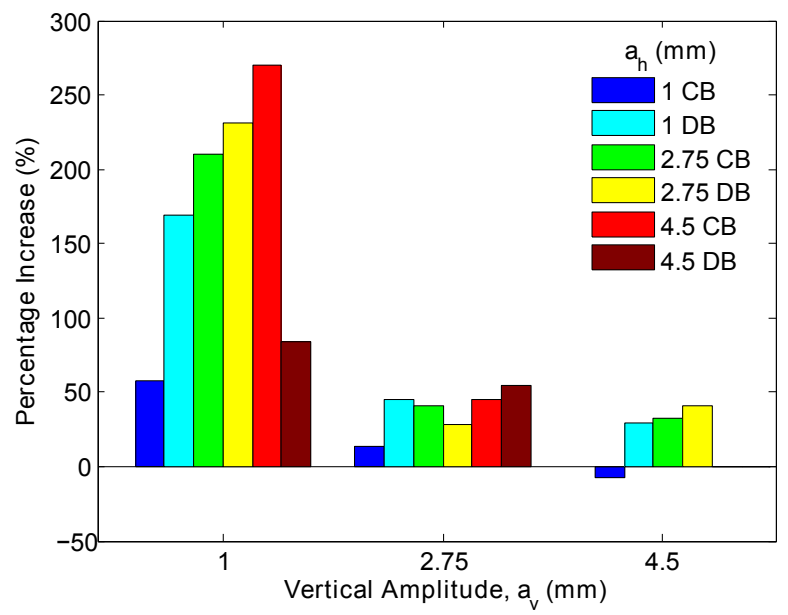

Figure 16: Bar graph showing the percentage gains of the CB and DB drilling depths from the simple motion depths.

drilling was established after the initial penetration. Given that the strength of the surrounding regolith can be considered to be much larger than the horizontal force provided by the mechanism, the drill is not able to push into the regolith, with the reaction force of the regolith instead pushing the mechanism back. It is proposed that the ideal motion of Figure 5 is instead split in two. This consists of the drill head moving sideways into the regolith a fraction of the total ideal distance, and a reaction movement in which the DCMM is pushed in the opposite direction. This split motion is shown in Figure 17 (c), and is compared to the ideal complex (b) and simple (c) motions.

Despite this revision of the mechanics, the principle remains the same, albeit with a reduced effect. The force applied by the mechanism will compress the regolith which, coupled with the slight movement of the drill heads to further engage it, creates a greater traction force, resulting in the increase in penetration. Stronger mechanisms, able to resist the regolith reaction, would allow deeper digging of the drill into the surrounding substrate, creating a motion closer to the ideal. This would further compress the regolith, creating an even greater traction force that would improve the penetration performance.

From these results, it can be concluded that the addition of controlled lateral movements presents a benefit to dual-reciprocating drilling by increasing the drilling depth. Increasing the amplitude of both the horizontal and 


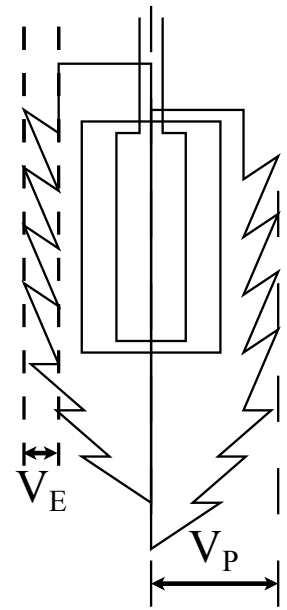

Simple motion

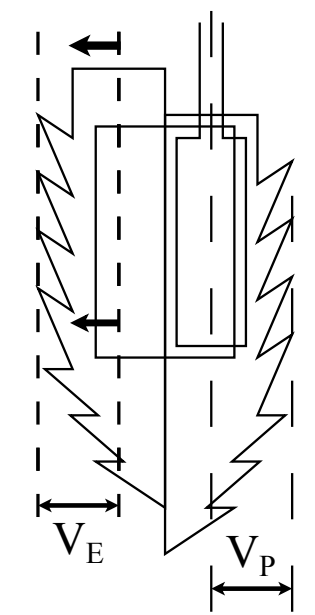

Ideal complex motion

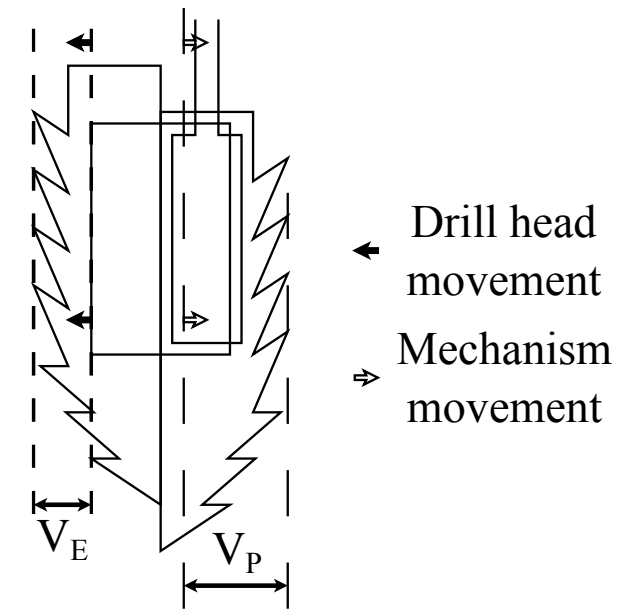

Actual complex motion

Figure 17: Comparison of the movement of the drill head halves and internal mechanism during regular simple motion, ideal complex motion and what is believed to have occurred during the experiments.

vertical reciprocations produces a greater increase in depth. Additionally, the depth gain created by these sideways movements is fairly constant for different vertical amplitudes, and there is also evidence that the diagonal burrowing method is slightly more effective than circular burrowing. Caution must be taken when increasing the amplitudes, as doing so also increases the regolith's resistance to the motion, producing a greater strain on the mechanism that increases the risk of the system jamming or parts failing. This was often experienced during the experiments, with frequent maintenance of the system required to ensure smooth operation for the larger amplitudes.

\section{Drilling at an Angle}

The phenomenon of drill stem bending causing a larger drilling depth, discussed in Section 2, was seen again during these tests. During the simple motion experiments, and in those of the first $\mathrm{CB}$ complex motion tests, with $a_{h}$ set at $1 \mathrm{~mm}$, the original holding part, shown in Figure 7 (c), was a single plastic piece. Its flexibility and loose connections allowed the mechanism to bend slightly, resulting in the drill penetrating at an angle. After the hold broke, a replacement made of stronger material was built, shown in Figure 7 
(a), greatly reducing the flexibility and resulting in vertical drilling. The experiments performed with the original hold were redone. A comparison of the depths achieved by the angled and vertical tests, given in Figure 18, show the same trends as the previous observations made with drill stem bending, with the depth achieved when drilling at an angle clearly being greater than when drilling vertically.

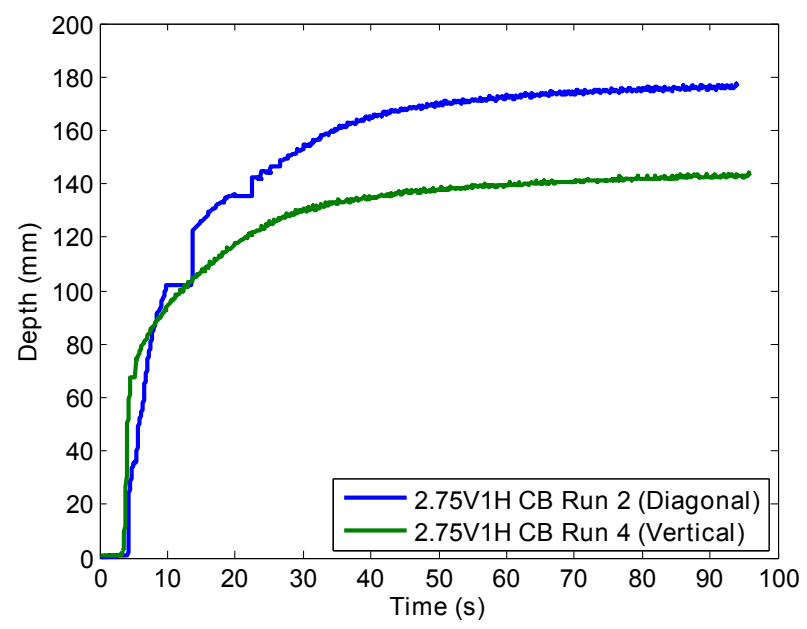

Figure 18: Comparison of the depth profiles of the 2.75V1H CB set-up when drilling straight and at an angle.

To examine this in controlled conditions, the test rig is tilted at an angle of $15^{\circ}$. An additional series of experiments using the simple and $2.75 \mathrm{~mm}$ $a_{h} \mathrm{CB}$ motions was performed, with the aim of providing clear evidence as to the depth gain created by controlled diagonal drilling. By this stage of the experiments general wear and tear of the mechanism, causing looser connections, resulted in the amplitudes being slightly reduced. Also, due to increasing levels of maintenance creating more unsuccessful runs and failures, the second $4.5 \mathrm{~V} 2.75 \mathrm{H}$ run was not performed. Despite these issues, it was still possible to gather enough meaningful data to make a number of observations. Given that the potentiometer used to measure the distances was also tilted along with the test rig, the values recorded were converted to give the vertical depths. These, alongside the corresponding depths found in the experiments performed with the vertical test rig, are given in Figure 19. From this graph it can be seen that, for all cases, the runs performed at the $15^{\circ}$ angle all achieved a greater depth than the vertical runs. This confirms the observations made 
in previous experiments, and it can be concluded that drilling at an angle appears to increase the drilling performance.

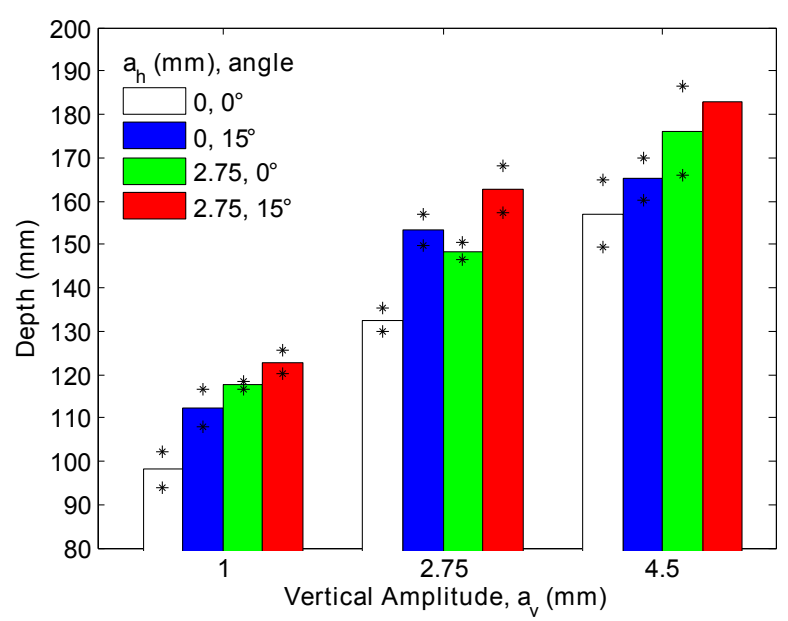

Figure 19: Graph comparing the depths achieved when drilling vertically and at an angle of $15^{\circ}$.

\subsection{Proposition of Diagonal Drilling Mechanics}

Information regarding drilling at an angle, or directional drilling, of regolith is scarce. Directional drilling of rocks is often used for the purpose of reaching targets otherwise inaccessible by conventional vertical drilling. Studies into directional drilling have generally focused on the deviation of vertical penetration caused by the geology and properties of the rocks being drilled into, and how such deviations can be accounted for and controlled (Bar-Cohen and Zacny, 2009). Investigations into diagonal drilling in regolith have so far been limited to inclined cone penetration tests, which largely examined the effects of inclination on tip resistance (Jiang et al, 2014).

The elastic behaviour of soils can be determined by the relationship between the effective horizontal and vertical stresses, $\sigma_{h}^{\prime}$ and $\sigma_{v}^{\prime}$. Changes in the soil properties, such as volume and shear strength, are governed by the effective stresses. The vertical stress of a soil element is determined by weight of the soil above it. This compresses the element vertically, but is prevented from expanding outwards by the horizontal stress (Terzaghi et al, 1996). The ratio of these stresses, known as the coefficient of earth pressure at rest, $K_{0}$, is defined by the soil's internal angle of friction, $\phi^{\prime}$, given in Equation (3). This equation is a suitable approximation for normally consolidated materials 
allowed to settle naturally under gravity, and gives a range of $0.31-0.67$ for clays and soils. Materials that have undergone some compression, through ageing or preshearing, will likely have an in-situ $K_{0}$ greater than that given by Equation (3), though still lower than 1 . Whilst $K_{0}$ can be higher than 1 , this is only achieved by significant compression of the soil from vibration and compaction methods, and as such the equations governing these conditions are not applicable for these experiments.

$$
K_{0}=\frac{\sigma_{h}^{\prime}}{\sigma_{v}^{\prime}} \quad \text { where } \quad K_{0}=1-\sin \left(\phi^{\prime}\right)
$$

A potential explanation for the increased depth experienced by diagonal drilling is that, given the typical range of values of $\mathrm{K}, \sigma_{h}^{\prime}$ is smaller than $\sigma_{v}^{\prime}$, and as such, compressing the regolith in a horizontal direction is easier than doing so in a vertical direction. As diagonal drilling involves penetration in both directions, taking both of these stresses into account means that the net effective stress will be lower than for vertical drilling at the same depth, as shown in Figure 20 .

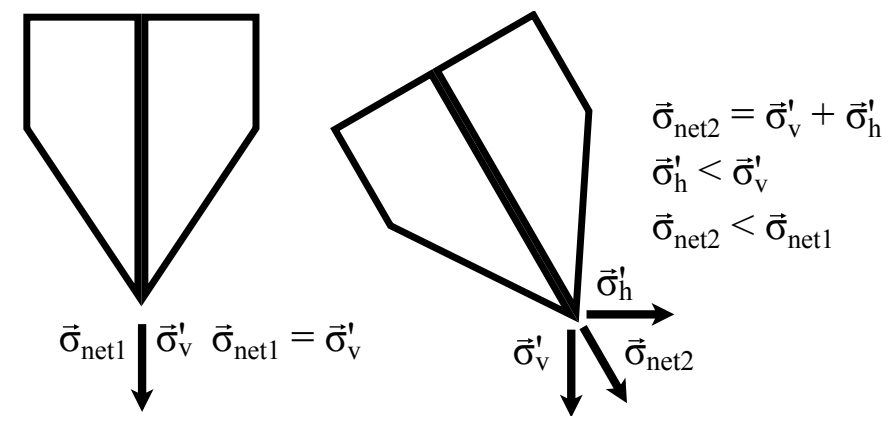

Figure 20: Diagram of the proposed effective stresses acting upon the vertical and diagonal drill.

Effective stress is not the only factor in determining penetration performance, with other variables such as deformation patterns also likely having an effect. However, this can potentially be used a simplified first explanation of the benefits of drilling regolith at an angle and presents an opportunity for this new drilling method to be studied in further detail. 


\section{Conclusions}

This paper has discussed the effects of the addition of controlled lateral movements to the drilling motion of the DRD. This has involved the design and construction of an integrated actuation mechanism able to achieve the original reciprocation and new complex motions. The Quadruple Cam design, selected from a mechanism concept trade-off study for its ability to produce the circular and diagonal burrowing motions, was constructed. A series of experiments were conducted with the drill deployed from a test rig, with the results allowing an examination of the forces present within the mechanism during reciprocation, and the determination and comparison of the depths achieved by a range of drilling motions.

Using an integrated force sensor, the profiles of the penetration and traction forces were determined, and were shown to be consistent during free running with varying amplitudes and operating conditions. It could be seen that the penetration forces experienced were consistently larger than the traction forces, and the magnitude of both increased with the final depth achieved. An analytical investigation of these forces revealed that the vast majority of the free-run forces acting on the sensor were as a result of the mechanism's internal friction, in particular due to the addition of the drill heads.

Analysis of the final depths achieved by each of the drilling motions revealed that, for every vertical amplitude, almost all experiments using the complex motion achieved a greater depth than those of the corresponding simple motions. Several trends could be seen; in particular the increasing depth achieved with greater horizontal amplitudes used, while the overall gains achieved with each $a_{h}$ remained approximately constant with changing $a_{v}$ values. This suggests that the depth gains of the sideways movements are not affected by the vertical reciprocation amplitude. This provides evidence suggesting that the lateral movements allow the teeth to further dig into the surrounding regolith, producing greater traction. Further examination of lateral movements was done by performing a number of experiments at a $15^{\circ}$ angle. These diagonal tests also resulted in greater depths than those performed vertically. These results have led to the proposition of the penetration mechanics from which the lateral movements and diagonal drilling create new interactions with the regolith that produce this improved performance.

These experiments have demonstrated both the first testing of an integrated actuation mechanism for the $\mathrm{DRD}$, including examination of the forces 
acting on the mechanism during drilling, as well as the creation of new burrowing motions with actively controlled lateral movements. The increased depths achieved by the complex motions and by drilling at an angle have shown how the penetration performance of conventional dual-reciprocating drilling can be improved. This can lead to new avenues of research into drilling motions and how lateral movements affect the mechanics of the soil and the interaction of the teeth. The next step in the development of the DRD will be to determine how best to integrate the lateral movements into the internal mechanism, leading to the development of a first completed system prototype.

\section{Acknowledgements}

The authors thank OHB System AG for providing funding for the $\mathrm{PhD}$ of which this paper will contribute to.

\section{References}

Badescu, M., Kassab, S., Sherrit, S., Aldrich, J., Bao, X., Bar-Cohen, Y., \& Chang, Z., 2007, Ultrasonic/Sonic Driller/Corer as a hammer-rotary drill, In Proceedings of Sensors and Smart Structures Technologies for Civil, Mechanical and Aerospace Systems, San Diego, USA.

Bansal, R., \& Brar, J., 2015, A Textbook of Theory of Machines, Laxmi Publications Ltd, 5th Edition.

Bar-Cohen, Y., \& Zacny, K., 2009, Drilling in Extreme Environments, Wiley$\mathrm{VCH}$.

Frame, T., 2012, Evolution of a New Planetary Drill Design Using Bioinspired Dual Reciprocating Drilling Technique, In 63rd International Astronautical Congress, Naples, Italy.

Gao, Y., Ellery, A., Jaddou, M., Vincent, J., \& Eckersley, S., 2007, Planetary Micro-Penetrator Concept Study with Biomimetic Drill and Sampler Design, IEEE Transactions on Aerospace and Electronic Systems, 43 (3), 875-885.

Gao, Y., Ellery, A., Sweeting, M., \& Vincent, J., 2007, Bioinspired Drill for Planetary Sampling: Literature Survey, Conceptual Design, and Feasibility Study, Journal of Spacecraft and Rockets, 44 (3), 703-709. 
Gao, Y., Frame, T. \& Pitcher, C., 2015, Piercing the Extraterrestrial Subsurface. Integrated Robotic Drill for Planetary Exploration, IEEE Robotics and Automation Magazine, 22 (1), 45-53.

Gouache, T., Gao, Y., Coste, P. \& Gourinat, Y., 2011a, First experimental investigation of dual-reciprocating drilling in planetary regoliths: Proposition of penetration mechanics, Planetary and Space Science, 59, 1529-1541.

Gouache, T., Gao, Y., Frame, T., Coste, P. \& Gourinat, Y., 2011b, Identification of the Forces Between Regolith and a Reciprocating Drill-Head: Perspectives for the Exploration of Martian Regolith, In 62nd International Astronautical Congress, Cape Town, South Africa.

Grygorczuk, J., Wiśniewski, Ł., Kędziora, B., Borys, M., Przybyła, R., Kuciński, T., Ossowski, M., Konior, W., Krömer, O., Spohn, T., Tokarz, M., Białek, M., 2016, Hammering Mechanism for HP3 Experiment (InSight), In Proceedings of 43rd Aerospace Mechanisms Symposium, Santa Clara, USA.

Jiang, M., Dai, Y., Cui, L., Shen, Z., \& Wang, X., 2014, Investigating mechanism of inclined CPT in granular ground using DEM, Granular Matter, 16 (5), 785-796.

Menon, C., Vincent, J., Lan, N., Bilhaut, L, Ellery, A., Gao, Y., Zangani, D., Carosio, S., Manning, C., Jaddou, M., \& Eckersley, S., 2006, BioInspired Micro-Drills for Future Planetary Exploration, In Proceedings of CANEUS, Toulouse, France.

Pitcher, C. \& Gao, Y., 2015, Analysis of drill head designs for dualreciprocating drilling technique in planetary regoliths, Advances in Space Research, 56, 1765-1776.

Pitcher, C., Gao, Y. \& Richter, L., 2015, Evolution of an Integrated Actuation Mechanism for Planetary Exploration Using Dual-Reciprocating Drilling, In 13th Symposium on Advanced Space Technologies in Robotics and Automation (ASTRA), ESA-ESTEC, Noordwijk, The Netherlands.

Re, E., Magnani, P., Izzo, M., Rizzi, F., Senese, S., del Campo, F., Finotello, R., Ferrario, R., 2008, ExoMars multi rod drill development and testing, In 10th Workshop on Advanced Space Technologies for Robotics and Automation, Noordwijk, The Netherlands. 
Scott, G., \$ Saaj, C., 2009, Measuring and Simulating the effect of variations in soil properties on microrover trafficability, In AIAA Space 2009 Conference and Exposition, Pasadena, USA.

Terzaghi, K., Peck, R., \& Mesri, G., 1996, Soil Mechanics in Engineering Practice, John Wiley \& Sons, Inc., 3rd Edition.

The Mechanism of Drilling by Wood Wasp Ovipositors, Biomimetics, 3 (4), 187-201. 